\title{
Identification of a stemness-related gene panel associated with BET inhibition in triple negative breast cancer
}

\author{
Leticia Serrano-Oviedo $^{1,2}$ - Miriam Nuncia-Cantarero ${ }^{1}$. Sara Morcillo-Garcia ${ }^{1}$. Cristina Nieto-Jimenez ${ }^{1,2}$. \\ Miguel Burgos ${ }^{2,3}$ • Veronica Corrales-Sanchez ${ }^{2}$ - Javier Perez-Peñaa ${ }^{1,2} \cdot$ Balázs Györffy $^{4}$. Alberto Ocaña ${ }^{1,2,3,5}$ (D) \\ Eva María Galán-Moya ${ }^{1,3}$ (i)
}

Accepted: 20 February 2020 /Published online: 12 March 2020

(C) The Author(s) 2020

\begin{abstract}
Purpose Triple negative breast cancers (TNBCs) are enriched in cells bearing stem-like features, i.e., cancer stem cells (CSCs), which underlie cancer progression. Thus, targeting stemness may be an interesting treatment approach. The epigenetic machinery is crucial for maintaining the stemness phenotype. Bromodomain and extra-terminal domain (BET) epigenetic reader family members are emerging as novel targets for cancer therapy, and have already shown preclinical effects in breast cancer. Here, we aimed to evaluate the effect of the BET inhibitor JQ1 on stemness in TNBC.

Methods Transcriptomic, functional annotation and qRT-PCR studies were performed on JQ1-exposed TNBC cells in culture. The results obtained were confirmed in spheroids and spheroid-derived tumours. In addition, limiting dilution, secondary and tertiary tumour sphere formation, matrigel invasion, immunofluorescence and flow cytometry assays were performed to evaluate the effect of JQ1 on CSC features. For clinical outcome analyses, the online tool Kaplan-Meier Plotter and an integrated response database were used.

Results We found that JQ1 modified the expression of stemness-related genes in two TNBC-derived cell lines, MDA-MB-231 and BT549. Among these changes, the $C D 44$ Antigen/CD24 Antigen $(C D 44 / C D 24)$ ratio and Aldehyde Dehydrogenase 1 Family Member A1 (ALDH1A1) expression level, i.e., both classical stemness markers, were found to be decreased by JQ1. Using a validated spheroid model to mimic the intrinsic characteristics of CSCs, we found that JQ1 decreased surface CD44 expression, inhibited self-renewal and invasion, and induced cell cycle arrest in $\mathrm{G} 0 / \mathrm{G} 1$, thereby altering the stemness phenotype. We also found associations between four of the identified stemness genes, Gap Junction Protein Alpha 1 (GJAl), CD24, Epithelial Adhesion Molecule (EPCAM) and SRY-related HMG-box gene 9 (SOX9), and a worse TNBC patient outcome. The expression of another two of the stemness-related genes was found to be decreased by JQ1, i.e., ATP Binding Cassette Subfamily G Member 2 (ABCG2) and RUNX2, and predicted a low response to chemotherapy in TNBC patients, which supports a role for $R U N X 2$ as a potential predictive marker for chemotherapy response in TNBC.

Conclusions We identified a stemness-related gene panel associated with JQ1 and describe how this inhibitor modifies the stemness landscape in TNBC. Therefore, we propose a novel role for JQ1 as a stemness-targeting drug. Loss of the stem cell
\end{abstract}

Leticia Serrano-Oviedo and Miriam Nuncia-Cantarero contributed equally to this work.

Electronic supplementary material The online version of this article (https://doi.org/10.1007/s13402-020-00497-6) contains supplementary material, which is available to authorized users.

Alberto Ocaña

alberto.ocana@salud.madrid.org

Eva María Galán-Moya

EvaMaria.Galan@uclm.es

1 Translational Oncology Laboratory, Centro Regional de Investigaciones Biomédicas (CRIB), Universidad de Castilla-La Mancha: Campus de Albacete, C/ Almansa 14, 02008 Albacete, Spain

2 Translational Research Unit, CIBERONC and Complejo Hospitalario Universitario de Albacete, Albacete, Spain
3 Physiology and Cell Dynamics Laboratory, Centro Regional de Investigaciones Biomédicas (CRIB), Universidad de Castilla-La Mancha: Campus de Albacete, Albacete, Spain

4 Semmelweis University 2nd Dept. of Pediatrics, and MTA TTK Lendület Cancer Biomarker Research Group, Institute of Enzymology, Hungarian Academy of Sciences, Budapest, Hungary

5 Experimental Therapeutics Unit, Medical Oncology Department, Hospital Clínico San Carlos and IdISSC, Madrid, Spain 
phenotype via JQ1 treatment could lead to less aggressive and more chemo-sensitive tumours, reflecting a better patient prognosis. Thus, the identified gene panel may be of interest for the clinical management of patients with aggressive TNBC.

Keywords Triple negative breast cancer $\cdot$ BET inhibitors $\cdot$ Biomarkers $\cdot$ Targeted therapy $\cdot$ Cancer stem cells $\cdot$ Tumour heterogeneity · Stemness

\section{Introduction}

Cancer progression involves loss of differentiation and acquisition of multipotency and asymmetric division characteristics [1]. Accordingly, some cancer cells adopt stem cell-like features, such as self-renewal and the ability to differentiate into multiple cell lineages from the tissue of origin [2]. This subpopulation of cells also shows an increased expression of stem cell-related genes [3] and, as such, they have been dubbed cancer stem cells (CSCs). CSCs participate in a number of processes during cancer progression, i.e., they are intrinsically resistant to standard chemotherapies, play a central role in cancer metastasis and appear to be responsible for tumour recurrence [4-6]. Together, these characteristics are known as cancer stemness [7].

Also, abnormal epigenetic gene regulation may contribute to cancer initiation and progression, and affect the stemness phenotype $[8,9]$. Through a deregulated epigenetic program, tumours can increase the number of CSCs, suggesting that the epigenetic machinery is essential for the maintenance of stem cell identity. Consequently, targeting epigenetic-related mechanisms may be a promising strategy to inhibit this cancer cell subpopulation [10]. Several epigenetic inhibitors are currently under clinical evaluation for cancer treatment, among which inhibitors of histone deacetylases (HDACs), enhancer of Zeste homolog 2 (EZH2), DNA methyltransferases (DNMTs), lysine-specific demethylase 1 (LSD1), and bromodomain and extra-terminal domain (BET) family proteins $[11,12]$. The BET family of proteins, including BRD2, BRD3, BRD4 and BRDT, plays a crucial role in epigenetic regulation of gene transcription through the ability to recognise histone acetylated lysine residues. These epigenetic readers have recently emerged as new therapeutic targets for cancer, as well as for metabolic and inflammatory diseases $[8,13,14]$. These epigenetic drugs exhibit antiproliferative effects through a complex mechanism that involves gene transcription inhibition, cell cycle arrest and, therefore, altered cell division. In addition, BET inhibitors (BETi) have shown preclinical activity in breast cancer [13-15], although, the use of BETi as single agents in clinical trials has so far yielded modest results [15].

Triple negative breast cancer (TNBC) is a subtype of breast cancer for which currently limited therapeutic options exist. This is because it lacks specific targets that can be used to guide therapy. Although aggressive chemotherapy can lead to an initial high response rate, TNBC patients often develop resistance to chemotherapy, and distant relapses are frequently observed
[16-18]. Consequently, effective treatment of TNBC remains an unmet need and the identification of targets to treat this subtype is essential for improved therapeutic efficacy. Resistance to chemotherapy usually occurs in CSCs, which exhibit a low proliferation rate [5]. Similarly, tumour relapses are associated with the presence of dormant CSCs [19]. As such CSCs are, at least partly, considered to be responsible for the high degree of therapy resistance in TNBC [20]. Therefore, targeting stemness may be a potential strategy to address this disease.

Here, through transcriptomic and functional annotation analyses, we identified a panel of stemness-related genes that can efficiently be targeted with the BETi JQ1. Moreover, we found that JQ1 impaired several CSC features in a three-dimensional (3D) model of TNBC, including growth under non-adherent conditions, self-renewal and expression of stemness marker genes. Importantly, we found that JQ1-mediated downregulation of this stemness signature was linked to improved TNBC patient prognosis, and that two of the genes identified, $A B C G 2$ and $R U N X 2$, have the ability to predict response to chemotherapy.

\section{Material and methods}

\subsection{Cell culture and drug compounds}

MDA-MB-231 and BT-549 TNBC cells were grown as adherent monolayers in Dulbecco's Modified Eagle's Medium (DMEM) containing 10\% Foetal Bovine Serum (FBS), $100 \mathrm{U} / \mathrm{ml}$ penicillin, $100 \mu \mathrm{g} / \mathrm{ml}$ streptomycin and $2 \mathrm{mM} \mathrm{L-}$ glutamine (all from Sigma Aldrich, Merck KGaA, Darmstadt, Germany), in a $5 \% \mathrm{CO}_{2}$ atmosphere at $37^{\circ} \mathrm{C}$.

Spheroid models were designed as follows: a monolayer of adherent MDA-MB-231 or BT-549 cells was extensively washed with Phosphate-Buffered Saline (PBS) and gently scraped to detach them from the plastic surface. Next, the cells were centrifuged at $900 \mathrm{rpm}$ for $5 \mathrm{~min}$ and transferred to nonadherent plates containing a CSC-defined medium (FBS-free Dulbecco's Modified Eagle's Medium Nutrient Mixture F-12 Ham, DMEM F-12) (Sigma Aldrich, Merck KGaA, Darmstadt, Germany) containing $100 \mathrm{U} / \mathrm{ml}$ penicillin, $100 \mu \mathrm{g} / \mathrm{ml}$ streptomycin, B27 supplement (Invitrogen, Thermo Fisher Scientific Inc.), EFG (20 ng/ml) and FGF (20 ng/ml) (Sigma Aldrich, Merck KGaA, Darmstadt, Germany) for at least 21 days to generate primary spheroids. The BETi JQ1 and OTX015 were purchased from Selleckchem company. 


\subsection{Transcriptomic, functional enrichment and gene expression analyses}

MDA-MB-231 cells were exposed to JQ1 (500 nM) for 12 and $24 \mathrm{~h}$. Next, RNA was isolated using a Qiagen RNeasy kit following the manufacturer's instructions. DNase treatment was performed on the RNA samples. Transcriptomic analyses were performed using an Affymetrix Human Transcriptome Array 2.0 at the Genomic Platform of the Cancer Institute of Salamanca. To identify cellular functions altered by JQ1 treatment, the differentially expressed genes were analysed using DAVID Bioinformatics Resources 6.7 (https://david.ncifcrf.gov/summary.jsp), a tool to perform gene set enrichment analyses. An adjusted $p$ value $<0.05$ was used to select the five highest ranked enriched gene-sets. Next, deregulated genes were classified according to their involvement in a wide range of cellular functions using Gene Set Enrichment Analysis (GSEA) software available at http:// software.broadinstitute.org/gsea/index.jsp. Among the potentially affected functions, we searched for those related to cancer stemness. This included all gene sets containing the words "differentiation" or "stem". The resulting list of JQ1-deregulated gene transcripts was then scrutinized to identify cancer stemness-related markers.

\subsection{Quantitative RT-PCR}

RNA isolation of all samples (MDA-MB-231 or BT-549 adherent, or spheroid cultures and MDA-MB-231 spheroidderived tumours) was performed using a RNeasy Mini kit (Qiagen), as indicated above. RNA concentrations and purities were determined using a NanoDrop ND-1000 spectrophotometer (Thermo Fisher Scientific Inc.). Next, $1 \mu \mathrm{g}$ of total RNA was reverse transcribed using a RevertAidHMinus First Strand cDNA synthesis kit (Thermo Fisher Scientific Inc.) in a thermocycler (Bio-Rad) under the following reaction conditions: $65{ }^{\circ} \mathrm{C}$ for $5 \mathrm{~min}, 42{ }^{\circ} \mathrm{C}$ for $60 \mathrm{~min}$, and $70{ }^{\circ} \mathrm{C}$ for $10 \mathrm{~min}$. The resulting cDNAs were subjected to quantitative real-time PCR (qRT-PCR) analysis using a Fast SYBR Green Master Mix (Thermo Fisher Scientific Inc.) in a StepOnePlus Real-Time PCR system (Applied Biosystems, Thermo Fisher Scientific Inc.). The conditions used included an initial step at $95{ }^{\circ} \mathrm{C}$ for $10 \mathrm{~min}$, followed by 40 cycles at $95{ }^{\circ} \mathrm{C}$ for $15 \mathrm{~s}$ and a final step at $60{ }^{\circ} \mathrm{C}$ for $1 \mathrm{~min}$. Each sample was analysed in triplicate, and cycle threshold $(\mathrm{Ct})$ values of transcripts were determined using StepOne Software v.2.1. Ct values were calculated using $G A P D H$ as reference. Untreated samples were used as controls to determine the relative fold-changes in messenger RNA (mRNA) expression. The primer sequences are listed in Supplementary Table 1.

\subsection{Immunofluorescence assays}

MDA-MB-231-derived spheroids were treated with JQ1 $(200 \mathrm{nM})$ and $72 \mathrm{~h}$ later floating spheroids were collected and gently dropped on a water repelling circle drawn on a poly-lysine-treated slide (DAKO). Within one minute, excess liquid was removed and slide-attached spheroids were fixed for 15 min with paraformaldehyde (4\%). After a PBS wash, samples were blocked with Bovine Serum Albumin (BSA) (5\%) for 30 min and, next, incubated with R-phycoerythrin (PE)-coupled CD44 Antigen (CD44) (R\&D Systems) for 60 min (3\% BSA). Extensive washes with PBS were performed before mounting with Fluoroshield (Sigma Aldrich, Thermo Fisher Scientific Inc.). 4,6-diamidino-2-phenylindole (DAPI) (Sigma Aldrich) was used for the staining of nuclei. Fluorescence imaging of spheroids was performed using confocal microscopy (Zeiss LSM 710).

\subsection{Limiting dilution and secondary and tertiary tumour sphere formation assays}

For the limiting dilation assays (LDA), MDA-MB-231 and BT-549 primary tumour spheres (TS), obtained from 21-day old spheroids, were mechanically disaggregated and counted before being seeded in non-adherent 96-well plates (Corning). Next, serial dilutions were performed to achieve 200 cells/well down to $1 \mathrm{cell} /$ well. Spheroid formation was monitored until day 21 by determining the number of TS per well.

For the secondary and tertiary TS formation assays, primary TS were mechanically dissociated after which 200.000 cells were cultured in ultralow attachment $100 \mathrm{~mm}$ plates (Falcon) in the presence of JQ1 $(200 \mathrm{nM})$ for 3 days. Next, the number of secondary TS per plate were determined. Secondary TS were again dissociated and left in the presence of the drug until day 6 , at which the number of tertiary TS per plate was determined. TS pictures were taken at the two time points using an inverted microscope (Nikon).

\subsection{Matrigel invasion assays}

Cells from freshly dissociated MDA-MB-231 and BT-549 spheroids $(10,000$ cells $)$ were seeded on a thin layer of matrigel in 48-well plates. After overnight incubation, the cells were exposed to JQ1 (200 nM), and 3 days later 3D invading structures were visualized under an inverted Nikon Eclipse TS1000 (20x) optical microscope.

\subsection{Flow cytometry assays}

For cell cycle analyses, MDA-MB-231 TS (500,000 cells) were cultured for $24 \mathrm{~h}$ before exposure to JQ1 (100 and $200 \mathrm{nM}$ ) for 1 day. Floating cells were collected and washed twice with cold PBS, fixed in ice-cold $70 \%$ ethanol for $30 \mathrm{~min}$ 
and, next, centrifuged at $5000 \mathrm{rpm}$ for $5 \mathrm{~min}$. The resulting cell pellets were washed in PBS containing 2\% BSA to prevent cell aggregation prior to incubation with propidium iodide (PI)/RNAse staining solution (Immunostep S.L., Salamanca, Spain) in the dark for $1 \mathrm{~h}$ at $4{ }^{\circ} \mathrm{C}$. Next, the samples were analysed on a FACSCanto II flow cytometer (BD Biosciences) and the percentage of cells in each cell cycle phase was determined by plotting spheroid DNA content against cell number using FACS Diva software.

For apoptosis evaluations, MDA-MB-231 TS $(300,000$ cells) were cultured for $24 \mathrm{~h}$ before exposure to JQ1 (100 and $200 \mathrm{nM}$ ). Three days later, floating cells were collected, centrifuged (900 rpm, $5 \mathrm{~min}$ ), and washed twice with ice-cold PBS before staining in $5 \mathrm{ml}$ Annexin V/DT-634 (Immunostep S.L.) in 1x binding buffer (10 mM HEPES, pH 7.4, $140 \mathrm{mM}$ $\mathrm{NaOH}, 2.5 \mathrm{mM} \mathrm{CaCl}_{2}$ ) for $1 \mathrm{~h}$ in the dark at room temperature. Viable cells, as indicated by negative Annexin V staining, were determined using a FACSCanto II flow cytometer (BD Biosciences).

\subsection{In vivo studies}

Balb/c nude mice (female, 6 weeks, $n=6$ /group) were orthotopically injected with freshly dissociated MDA-MB231-derived spheroids $\left(2 \times 10^{6}\right.$ cells). 14 days later, when the tumours reached $\sim 250 \mathrm{~mm}^{3}$ (Tumour volume $=$ (length $\mathrm{x}$ width $\left.^{2}\right) / 2$ ), the mice were treated daily for three days with JQ1 $(50 \mathrm{mg} / \mathrm{kg}$, intravenously). Next, the tumours were harvested and cryogenically stored until qRT-PCR analyses were performed (see above). All animal studies were carried out according to protocols approved by the ethics committee on animal experimentation of Castilla-La Mancha University (Procedure PR-2017-03-07).

\subsection{Clinical outcome analyses}

The Kaplan-Meier (KM) Plotter Online Tool was used to analyse relationships between the expression of JQ1-deregulated stemness genes and TNBC patient clinical outcome (http:// kmplot.com/analysis/, Relapse Free Survival (RFS), basal-like breast cancer; data accessed: 11/04/18). By using this tool, the effect of 54,675 genes on survival was assessed using 5143 breast cancer samples. For the analyses, patients were separated according to best cut-off values (expression range of the signature $=170-11,816$ ), and the following probes were used: Epithelial Adhesion Molecule (EPCAM) (201839_s at), SRYrelated HMG-box gene 9 (SOX9) (202936 s at), Integrin $\alpha 6$ (ITGA6) (201656 at), Gap Junction Protein Alpha 1 (GJA1) (201667 at), V-Myc avian myelocytomatosis viral oncogene homolog (MYC) (202431_s_at), ABCG2 (209735_at), EZH2 (203358 s at), Runt-related transcription factor 2 (RUNX2) (232231 at), Follistatin Like 1 (FSTL1) (208782 at), Protein Inhibitor of Activated STAT 3 (PIAS3) (203035_s_at),
Aldehyde Dehydrogenase 1 Family Member A1 (ALDH1A1) (212224 at), CD24 Antigen (CD24) (266 s at), and CD44 (212063 at). Only deregulated genes significantly associated with detrimental outcome (Hazard Ratio (HR) $>1$ and $p$ value $\leq 0.05)$ were used for subsequent analyses $(n=4)$. This tool was also used to determine RFS in combined analyses of the four poor prognosis-linked genes. All the analyses were performed independently by two authors (SMG and MNC) and reviewed by a third author (EMGM). No discrepancies were noted.

We established a database of transcriptomic datasets with available response and treatment data to study the relationship between JQ1-deregulated stemness gene expression and clinical outcome (RFS) regarding the chemotherapy regimen followed (5 years post-treatment). Through a Pubmed search using the keywords "breast cancer", "survival", "treatment", and "response", we identified 2108 breast cancer cases that received chemotherapy and for whom the gene expression levels were measured using Affymetrix HGU133A and HGU133A plus 2.0 microarrays. Gene expression levels were studied for patients who received chemotherapy with Taxane, Anthracycline, Ixabepilone, CMF (Cyclophosphamide / Methotrexate / Fluorouracil), FAC (Fluorouracil / Adriamycin / Cytoxan), and FEC (Fluorouracil / Epidoxorubicin / Cyclophosphamide).

\subsection{BETi-associated stemness interactome analysis}

The online tool STRING (http://www.string-db.org) was used to construct an interactome map of JQ1 stemness deregulated genes (STRING v10, data accessed: 14/03/18). Node degree: average number of interactions; Clustering coefficient: indicates the tendency of the network to form clusters. The closer the local clustering coefficient is to 1 , the more likely it is for the network to form clusters; PPI enrichment $p$ value: indicates the statistical significance.

\subsection{Statistical analysis}

All in vitro experiments were performed at least three times and, except for the flow cytometry studies, each condition was prepared in triplicate. Two-way ANOVA and Student's test were used for the statistical analyses $\left(p<0.05^{*}, p<0.01^{* *}\right.$, $\left.p<0.001^{* * *}\right)$.

\section{Results}

\subsection{JQ1 modifies the expression of stemness-related genes}

First, molecular functions modified by BET inhibition were explored at the transcriptomic level, with a special focus on those related to stemness. To this end, MDA-MB-231 cells were treated with JQ1 for 12 or $24 \mathrm{~h}$. Next, mRNA profiling 
analysis was performed using an Affymetrix Transcriptome Array 2.0. In total 4652 and 2530 deregulated transcripts were found at 12 and $24 \mathrm{~h}$, respectively. A preliminary analysis performed with the functional annotation tool DAVID revealed "Regulation of cell differentiation" as one of most ranked deregulated functions upon JQ1 treatment (Supplementary Fig. 1). Further functional enrichment analyses of the latter time point using GSEA software confirmed a decrease in several stem-related functions, including cell differentiation, stem cell differentiation, mesenchymal differentiation, regulation of cell differentiation, and endothelial cell differentiation (Fig. 1a and Supplementary Table 2).

Next, the list of deregulated transcripts was screened for genes known to be directly involved in stemness. This search uncovered a panel of 13 stem-related deregulated genes at $12 \mathrm{~h}$ and 13 genes at $24 \mathrm{~h}$ (Fig. 1b). The microarray-based results were corroborated by qRT-PCR, and we found that even low doses of JQ1 had an impact on the mRNA expression levels of the identified genes (Fig. 1c-g). To confirm its effect on the CSC population, the $C D 44 / C D 24$ ratio and the expression of ALDH1A1, both well-known CSC markers [21], were examined. We found that treatment with JQ1 induced decreases in the $C D 44 / C D 24$ ratio (Fig. 1c and g) together with a significant reduction in ALDH1A1 expression levels (Fig. 1d and g). Additionally, downregulation of the stemness-related genes $A B C G 2, I T G A 6, M Y C, E P C A M$ and $E Z H 2$ (Fig. 1e) and other well-known stemness-associated genes, such as GJA1, RUNX2, SOX9 and FSTL1, identified in the microarray screen, were confirmed to be downregulated upon JQ1 exposure by qRT-PCR, together with an increase in expression of the stemness repressor PIAS3 (Fig. 1f). These data confirm the impact of JQ1 on cancer stemness and identify a panel of stemness genes associated with its application.

\subsection{BET inhibition impairs stem cell-like features in TNBC spheroids}

Previously, three-dimensional cell culture models have been shown to be of use for cancer research [22]. As a general rule these models, grown under particular conditions, are enriched in stem cell markers, turning them into good models to explore the effects of BETi on stemness. Thus, to further confirm that MDA-MB-231 and BT-549 spheroids can be employed as in vitro models for CSCs, the basal expression of the genes identified in the transcriptomic analyses were compared in the spheroids and the parental adherent cells. Marked increases in the mRNA levels of these stemness markers were observed in the spheroid cultures (Fig. 2a-b). Of note, in comparison to the adherent cells, the $C D 44 / C D 24$ ratio was increased in the two spheroid models, being more than ten-fold in the MDA-MB231 spheroid model $(C D 44 / C D 24$ ratio $=11.48)$. Notably, ALDH1A expression was also augmented in both 3D models, especially in the BT549 spheroid model. The expression levels of other stem cell markers, such as Kuppel Like Factor 4 (KLF4) and SRY-Box2 (SOX2), were also found to be enhanced in the spheroid models (Fig. 2a).

Next, the effect of JQ1 on spheroid growth was explored. We found that exposure to JQ1 provoked a loss of the ability to grow in suspension, leading to the attachment of the spheroids to the culture plate surface, an indication of loss of stemness (Fig. 2c). Similar results were obtained with the BETi OTX-015 (Fig. 2c). The remaining spheroids were assessed for CD44 expression by immunofluorescence. We found that JQ1 induced a marked decrease in CD44 expression (Fig. 2d). To next evaluate the impact of JQ1 on MDAMB-231 and BT-549 spheroid self-renewal capabilities, a limiting dilution assay (LDA) in the presence of the drug was carried out. In support of a negative effect of JQ1 on stemness, cells exposed to JQ1 exhibited a reduced capacity to form primary spheres (Fig. 2e). To further investigate its influence on this stemness hallmark, secondary and tertiary TS formation assays were performed. We found that treatment with JQ1 drastically reduced the number and size of the spheres in both models (Fig. 2f). Another stem cell-like feature, i.e., invasion potential, was investigated via $3 \mathrm{D}$ matrigel assays using MDA-MB-231 and BT-549 spheroids. In agreement with the previous results, we found that JQ1 impaired the invasive ability of spheroids, resulting in a decrease of the area of the 3D structures formed and a reduction of the number of ramifications (Fig. 2g). Finally, in line with previous results in MDA-MB-231 adherent cells [12], cell cycle analyses by flow cytometry revealed that JQ1 also induced G0/G1 arrest in spheroids (Fig. 2h), without any impact on cell death (Fig. 2i).

\subsection{Expression of stemness-related genes in JQ1-treated spheroids and xenograft tumours}

Next, we aimed to confirm the microarray-based findings using validated CSC-mimicking models. To this end, the effect of JQ1 on the expression of the identified stem cell-like and CSC-like genes was investigated in MDA-MB-231 and BT-549 spheroids. First, the impact of JQ1 on CD44, CD24 and $A L D H 1 A 1$ expression was evaluated. JQ1 treatment led to a marked decrease of the $C D 44 / C D 24$ ratio $(0.74 \pm 0.13$ and $0.68 \pm 0.03$ for 100 and $200 \mathrm{nM}$, respectively, in the MDAMB-231 model, and $0.71 \pm 0.09$ for $200 \mathrm{nM}$ in the BT-549 model), together with a dramatic decrease in ALDH1A1 expression (Fig. 3d). The level of the stem cell marker GJA1, which is known to be enriched in CD44+ populations, was also reduced upon JQ1 exposure in the MDA-MB-231 model (Fig. 3a). Treatment with JQ1 efficiently downregulated the expression of the established CSC markers EPCAM, RUNX 2 and ITGA6, as well as the levels of other CSC-related genes, such as MYC, EZH2, FSTL1 and ABCG2 (Fig. 3b). The BETi also decreased the mRNA expression levels of the identified 
a

Stem Cell Differentiation

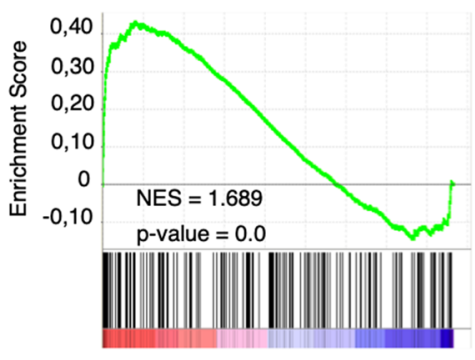

Control

JQ1 24h

Regulation of Cell Differentiation

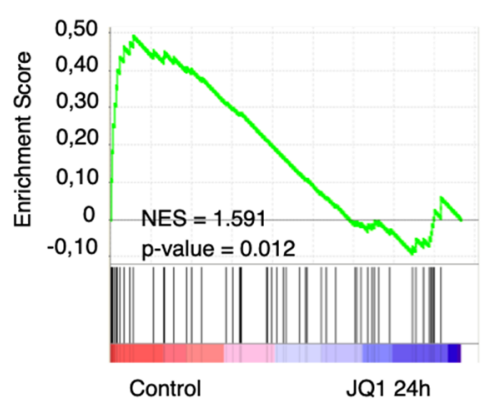

Messenchymal Differentiation

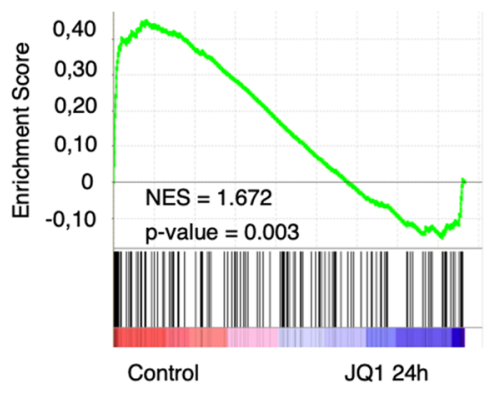

Endothelial Cell Differentiation
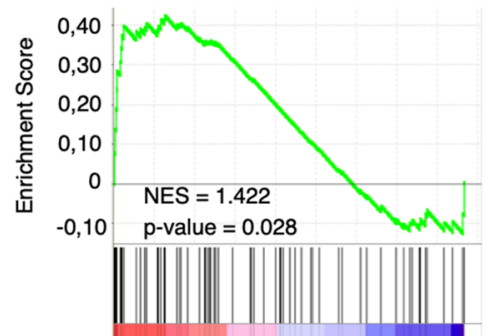

Control

JQ1 24h b

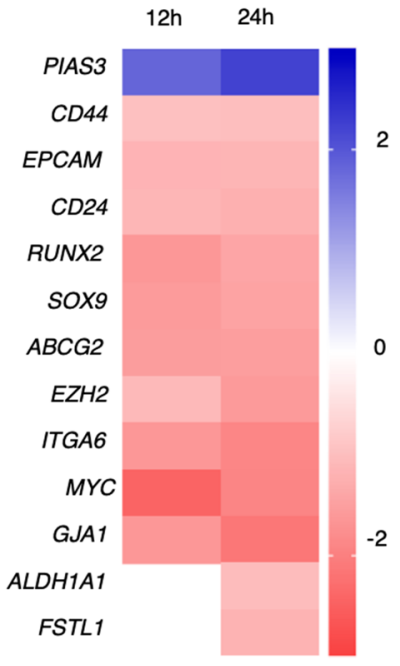

d

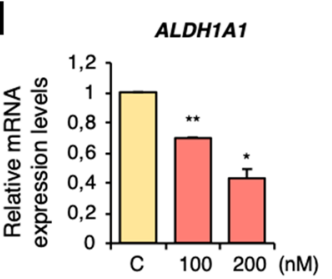

C

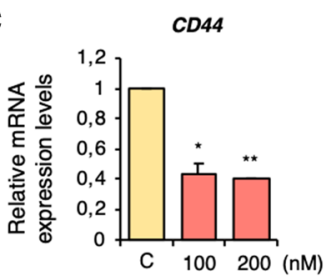

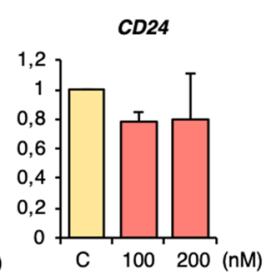

e
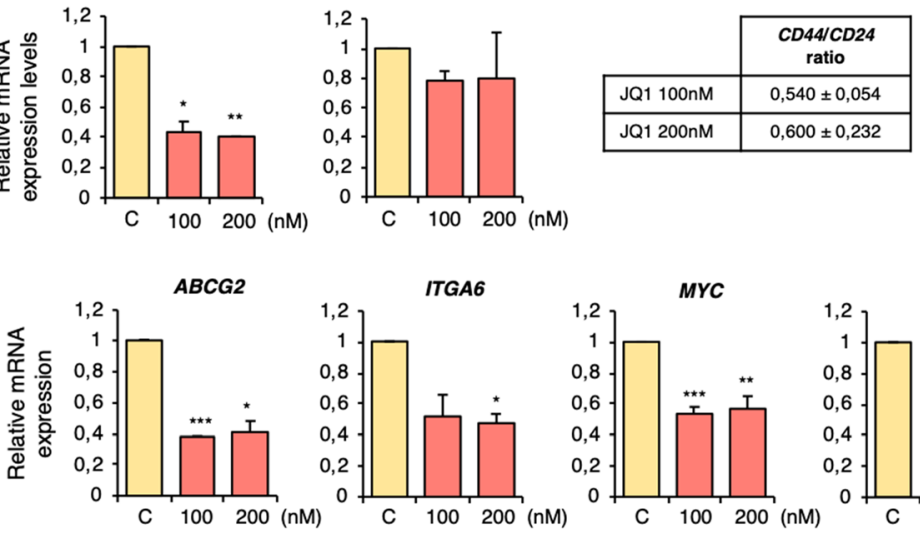

f
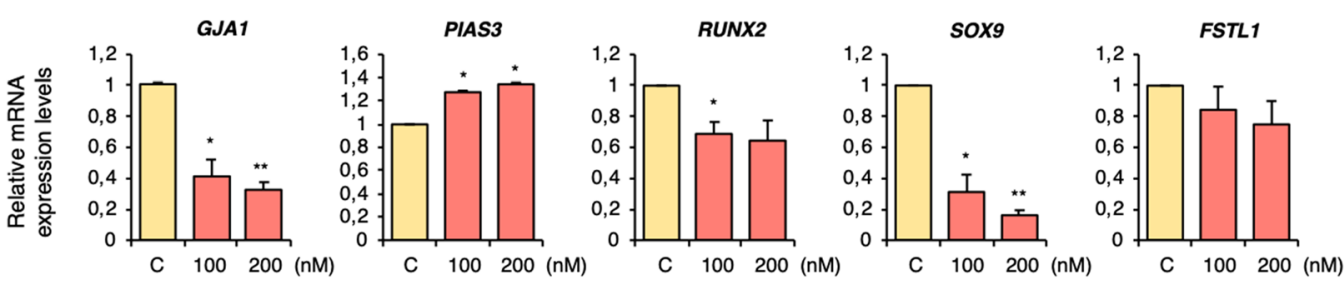

g
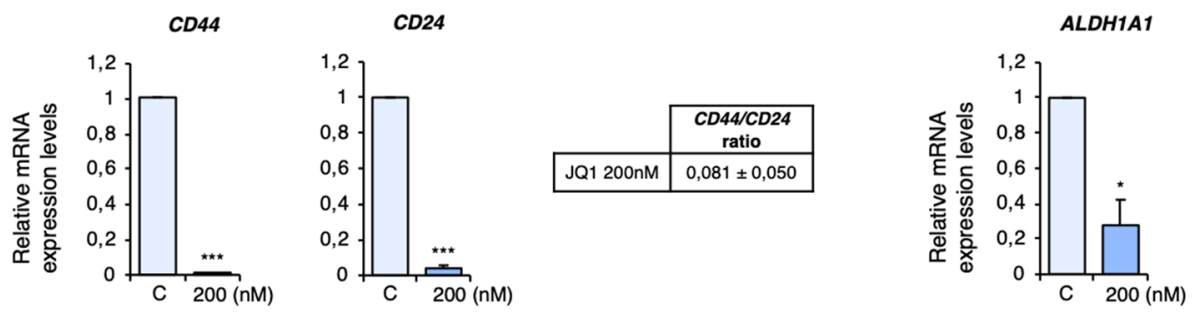
Fig. 1 JQ1 affects stemness-related functions and downregulates the expression of stemness-related genes. a. Gene Set Enrichment Analysis (GSEA) identifying stemness-related biological functions. b. Manual screening uncovering 13 stemness-related genes. Heat map indicating fold changes of JQ1-deregulated genes compared to those in nontreated cells (incubated with vehicle). c-g. JQ1 effect on mRNA levels of the stemness-related genes identified in $\mathbf{b}$ corroborated by qRT-PCR. MDA-MB-231 (c-f) or BT549 (g) cells were treated with the indicated doses of JQ1 (24 h) after which qRT-PCR was performed for the indicated genes. c and g. CD44 and CD24 mRNA levels were used to calculate the $C D 44 / C D 24$ ratios in JQ1 exposed cells compared to control cells. d and g ALDH1A1 mRNA levels were significantly downregulated in cells treated with JQ1 at both doses. e. JQ1 significantly decreased $A B C G 2$, ITGA6, MYC and EPCAM levels in MDA-MB-231 cells, and modestly affected the EZH2 level. f. GJA1, PIAS3, RUNX2 and SOX9 mRNA levels were significantly altered by JQ1, while just a slight decrease was found for FSTL1. The qRT-PCR conditions are described in material and methods. The results shown are averages of 3 independent experiments performed in triplicate. Student t-test: $* p<0,05$, ** $p<0,01$ and $* * * p<0,001$

TS-enriched classical stemness markers SOX2 and KLF4 (Fig. $3 c)$.

To test the potential of JQ1 to control the identified stemness-related gene panel in a more physiological context, its effect on MDA-MB-231 spheroid-derived xenografts was evaluated. First, Balb/c nude mice (female, 6 weeks) were injected with freshly dissociated MDA-MB-231 spheroids. Two weeks later, tumour-bearing mice were treated with JQ1 during three consecutive days. Having completed the treatment regimen, the mRNA levels of the identified panel of genes were analysed in the collected tumours. Our results revealed that JQ1 also affects the expression of $M Y C, A B C G 2$, ITGA6, EPCAM, SOX9, EZH2, GJA1, FSTL1 and SOX2 in this in vivo model (Fig. 4a). It had also a negative effect on the expression of CD24, CD44, ALDH1A, KLF4 and RUNX2 (Fig. 4b), further supporting a potential role for this BETi to alter stemness.

\subsection{JQ1-associated stemness signature predicts a worse patient outcome}

To evaluate the role of the identified genes in tumour relapse, we decided to study their association with clinical outcome in TNBC. Using data contained in the Kaplan Meier (KM) plotter online tool [23], we found that high expression levels of GJA1, CD24, EPCAM and SOX9 correlated with a poor recurrence free survival $(\mathrm{RSF})(\mathrm{HR}=1.55(1.21-2), p$ value $=$ $0.00057 ; \mathrm{HR}=1.5(1.14-1.97), p$ value $=0.0034 ; \mathrm{HR}=1.41$ $(1.09-1.83), p$ value $=0.0089 ; \mathrm{HR}=1.41(1.09-1.81), p$ value $=0.0075$, respectively) (Fig. 5a). Combined analysis of these four poor prognosis-associated genes revealed a higher potential to predict patient outcome than each gene individually $(\mathrm{HR}=1.85$ (1.35-2.53), log-rank $p=8.6 \mathrm{e}-05)$ (Fig. 5b). No association with clinical outcome was observed for the remaining genes.
Next, potential correlations between the identified genes and patient responses to antitumor therapy were assessed. To do so, correlations between responses to therapy and gene expression in over 2108 patient samples were determined. High mRNA levels of the JQ1-altered stemness-related genes $A B C G 2$ and $R U N X 2$ were found to correlate with poorer responses to chemotherapy (Fig. 5c, Supplementary Fig. 2). Specifically, high levels of RUNX2 expression were associated with low responses to any chemotherapy (Taxane, Anthracycline, Ixabepilone, CMF (Cyclophosphamide / Methotrexate / Fluorouracil), FAC (Fluorouracil / Adriamycin / Cytoxan), and FEC (Fluorouracil / Epidoxorubicin / Cyclophosphamide). On the other hand $A B C G$ 2, a well-studied multi-drug resistance-associated gene [24], showed correlations with only two chemotherapies (Taxane and Anthracycline). Therefore, RUNX2 may serve as a universal biomarker to predict chemotherapy response.

\section{Discussion}

The stemness landscape may define tumour aggressiveness through its effect on tumour progression and response to treatment. Accordingly, major interest in the study and measurement of stemness characteristics in cancer has recently evolved [8, 25] aimed at the development of novel strategies to target this feature. In breast cancer, stemness is a common characteristic that has been strongly associated with molecular and clinical features, especially in the TNBC subtype [9]. Indeed, TNBC is characterized by an enriched population of CSCs that confer intrinsic therapy resistance and aggressiveness $[4,20]$. Therefore, the identification of drugs targeting CSCs may be a promising approach to treat TNBC.

BETi have recently emerged as novel epigenetic cancer treatment options, exhibiting pre-clinical efficacy in several malignancies, including acute myeloid leukaemia and breast cancer $[15,26]$. In early stage clinical studies, good tolerability and signs of clinical activity for some of these compounds have been shown [15]. Among the different mechanisms of action, BETi have been found to play a role as regulators of self-renewal and stem cell signalling in some cancers, including medulloblastoma and head and neck squamous cell carcinoma [27, 28]. Although several studies have investigated the impact of BET protein inhibition in breast cancer [29-33], some of them evaluating its impact on epithelial-to-mesenchymal transition [34], little is known about the impact of their associated epigenetic drugs on cancer stemness in this cancer type. Here, we show how the BETi JQ1 alters the genetic stemness landscape, together with its associated features, and identify a stemnessrelated gene panel associated with BET inhibition in TNBC. By using different parameters to evaluate biomarkers of cancer stemness, such as overexpression of 


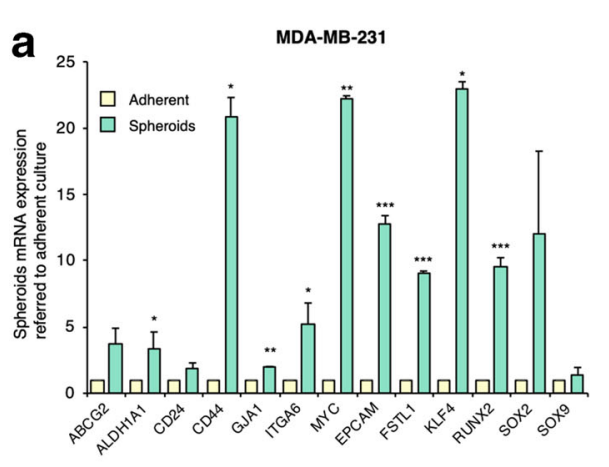

\section{b}
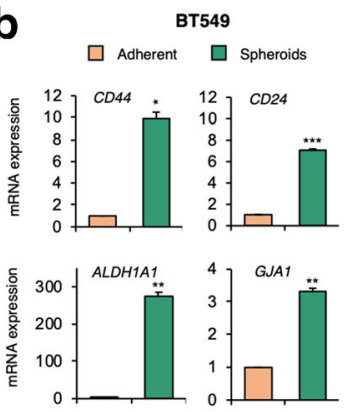

d

d

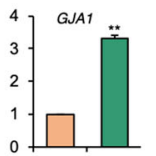

C
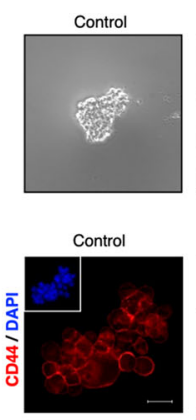

JQ1 (200 nM)

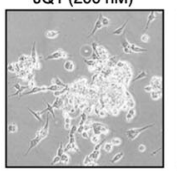

OTX-015 (400 nM)

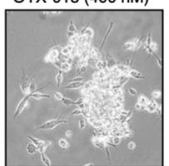

JQ1 (200 nM)

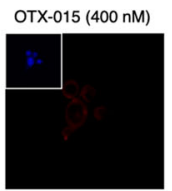

e

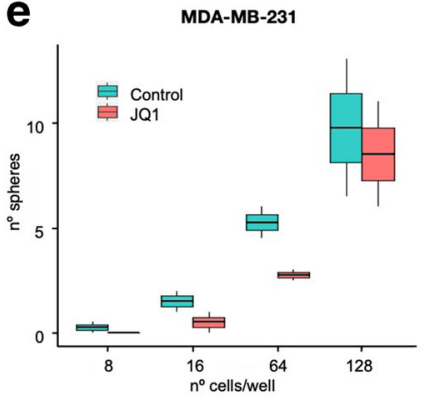

g
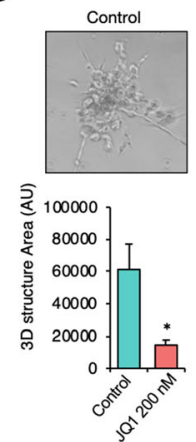

MDA-MB-231
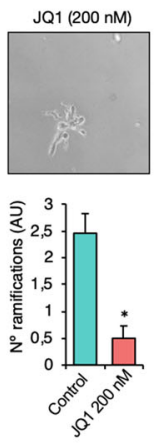
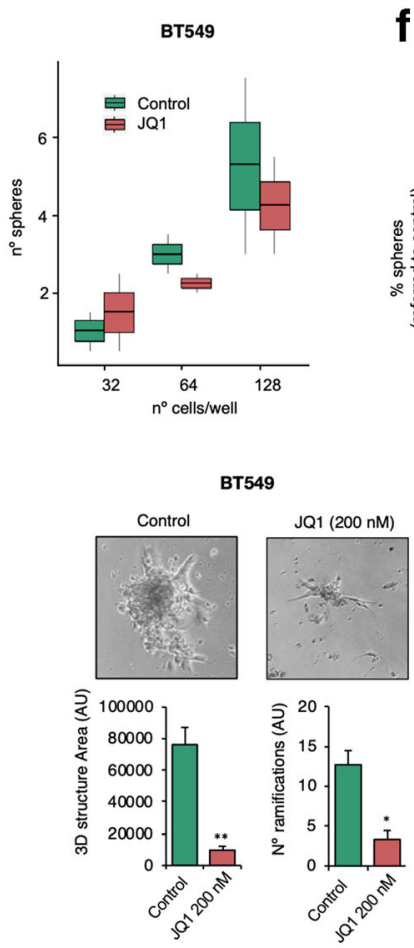

f

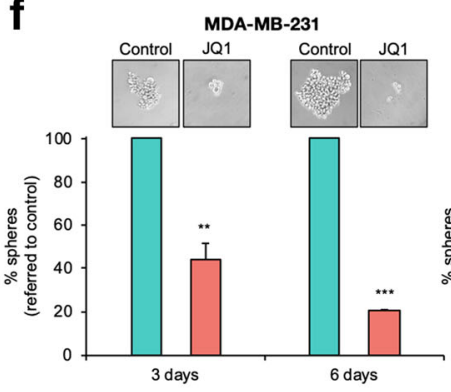

h

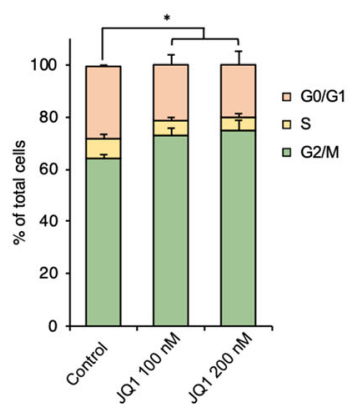

Fig. 2 BET inhibitor JQ1 impairs crucial stemness-related functions in CSC-mimicking spheroid models. a. Relative mRNA expression levels of several CSC markers in the MDA-MB-231 spheroid model (green) compared to those in adherent cultures (light yellow). b. Relative mRNA expression levels of $C D 44, C D 24, A L D H 1 A 1$ and GJA1 in the BT-549 spheroid model (dark green) compared to those in adherent cultures (orange). c. MDA-MB-231 cells were treated with the JQ1 or OTX-015 at the indicated doses for $72 \mathrm{~h}$ after which images were taken using an inverted microscope. $\mathbf{d}$. Remaining floating spheres from $\mathbf{c}$ were tested for CD44 surface expression (Red). Stained spheroids were observed by confocal microscopy. Squares display TS nuclear counterstaining with DAPI (blue). e. MDA-MB-231 and BT549 spheroids were mechanically dissociated and next exposed to JQ1 $(200 \mathrm{nM})$. Secondary formed spheroids were counted at day 3 and, subsequently, dissociated again to produced tertiary spheroids (day 6). Pictures were taken at both time points using and inverted microscope. f. MDA-MB-231 and BT549 spheroids were mechanically dissociated after which serial dilutions were performed to cover a range from 1 to 200 cells per well (in triplicates).

CSC markers, elevated CD44/CD24 ratios, self-renewal capabilities and high invasion potentials, we found that JQ1 can jeopardize stemness in TNBC.
LDA in the absence and presence of JQ1 $(200 \mathrm{nM})$ was performed to evaluate the self-renewal capacity of the spheroid models. The ability to form tumour spheres in both conditions was evaluated at day 21 and formed spheroids were counted. g. Freshly dissociated MDA-MB-231 or BT549 spheroids (10.000 cells/well in 48 -well plates) were gently layered on a thin matrigel matrix. $24 \mathrm{~h}$ later, matrigel-embedded cells were left untreated or exposed to JQ1 $(200 \mathrm{nM}) .48 \mathrm{~h}$ later, pictures of the invasion structures were taken, and $3 \mathrm{D}$ structure areas and the number of ramifications of each condition were evaluated. h. MDA-MB-231 spheroids were incubated with JQ1 at the indicated doses. After $24 \mathrm{~h}$, cell cycle progression was examined by flow cytometry. DNA staining was performed using propidium iodide. The histogram shows the percentage of cells in the different phases of the cell cycle. i. MDA-MB-231 spheroids were exposed to the indicated doses of JQ1. After $72 \mathrm{~h}$, apoptotic activity was assessed by flow cytometry by Annexin V binding evaluation. The histogram shows the percentage of viable cells (Annexin V-). All results shown represent the average of 3 independent experiments. Student t-test: $* p<0,05, * * p<0,01$ and $* * * p<0,001$

First, our transcriptomic analysis and qRT-PCR results in 2D, 3D and in vivo models, revealed several JQ1-associated stemness-related genes in TNBC, including CD44, CD24 and 

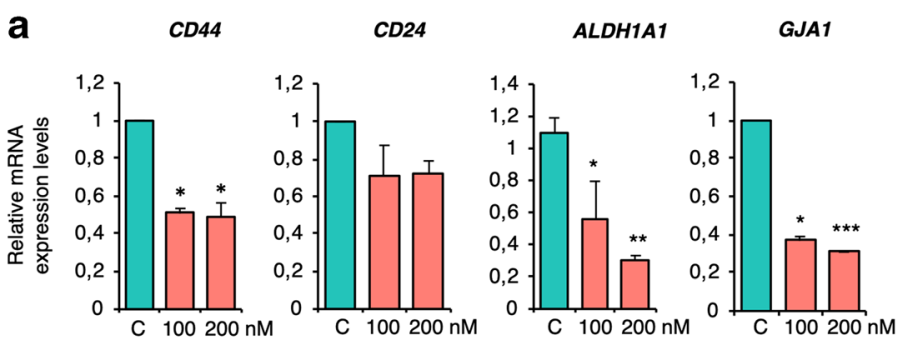

b

EPCAM
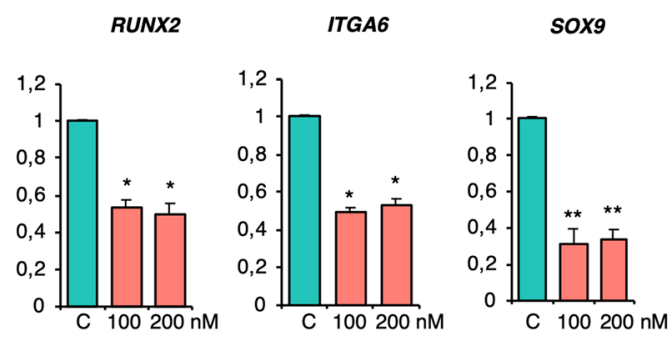

MYC
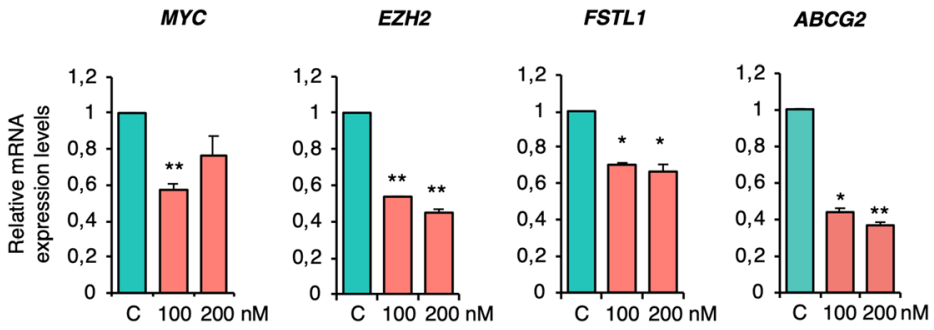

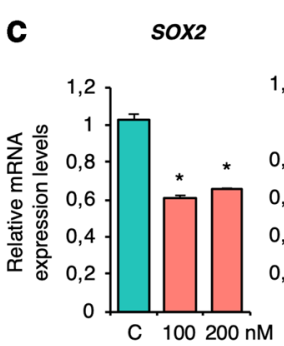

KLF4

d

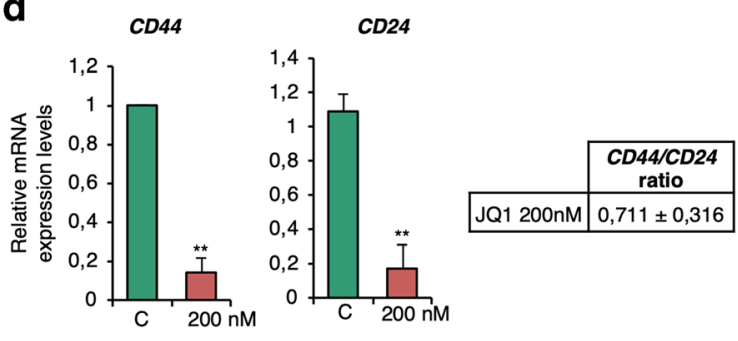

ALDH1A1

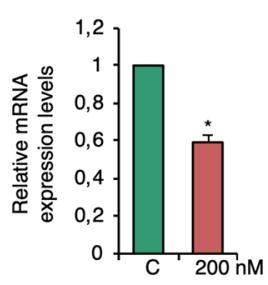

Fig. 3 JQ1 efficiently decreases the expression of CSC markers in MDAMB-231 (a) and BT549 (b) spheroids. c. Effect of JQ1 on the classical stemness markers SOX2 and KLF4. d. Relative mRNA levels of CD44, $C D 24$ and ALDH1A in spheroids exposed to JQ1 in MDA-MB-231 and
BT549 spheroids, respectively. The results shown represent the average of 3 independent experiments performed in triplicate. Student t-test: $* p<$ $0,05, * * p<0,01$ and $* * * p<0,001$
$A L D H 1 A 1$. The $C D 44 / C D 24$ ratio, as well as $A L D H 1 A 1$ overexpression, are known indicators of tumour aggressiveness, correlating with high proliferation indexes, increased tumour initiation capabilities and augmented invasion and metastasis potentials [21]. We found that JQ1 led to an almost $50 \%$ decrease in the $C D 44 / C D 24$ ratio and an up to $70 \%$ decrease in ALDH1A1 expression in TNBC cells. We also found that JQ1 negatively affected several transcription factors known to be involved in the initiation and maintenance of the stemness phenotype in TNBC, such as MYC, EZH2, SOX9 and RUNX2 [35-37]. MYC, EZH2 and SOX9 are known to be repressed in response to BET inhibition in several malignancies, such as medulloblastoma, bladder cancer and myelofibrosis [14, 38-40], but as yet the effect of JQ1 on these genes in TNBC has not been explored. Moreover, we found that $R U N X 2$, a stemness marker highly expressed in TNBC [41] and known to play a crucial role in breast cancer metastasis [42], can be negatively targeted by BETi in TNBC. This result, which is supported by previous data [43], could open the way to the development of RUNX2-targeting therapies. On the other hand, JQ1 may deregulate stemness genes that code for proteins involved in several other functions, such as EPCAM, ABCG2, ITGA6, GJA1, FSTL1 and PIAS3.
These proteins have been linked to cancer progression, resistance to chemo- and radiotherapy, poor prognosis and/or metastasis [4, 44-51]. Therefore, targeting these proteins with JQ1 could be of clinical interest in TNBC.

Two-dimensional cell culture models of cancer have progressively lost support over the last few years in favour of 3D models, which better reflect the in situ behaviour of cancer cells [22]. Several tools have been used to develop in vitro 3D cultures that mimic stemness features and are capable of predicting toxicity and resistance to anti-tumour compounds. Here, we used a MDA-MB-231-derived spheroid model to recapitulate TNBC and demonstrate that JQ1 not only modulates stemness-related genes in this $3 \mathrm{D}$ model, but also represses tumour self-renewal and invasion potentials, which are crucial stemness characteristics. JQ1 treatment did not lead to apoptosis in this population, but instead induced an increase in the $\mathrm{G} 0 / \mathrm{G} 1$ population, indicative of cell cycle arrest in this phase. More importantly, we found that his BETi directly impacted tumour sphere formation, a key stemness feature, as indicated by secondary and tertiary sphere formation and limiting dilution assays. This result points to a direct role of JQ1 as a tumour stemness regulator. 
MYC

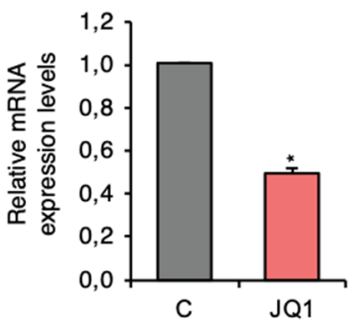

EPCAM

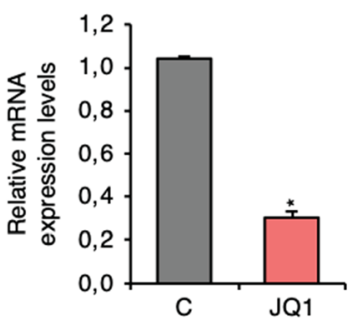

GJA1

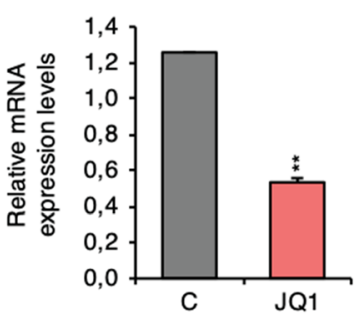

ABCG2

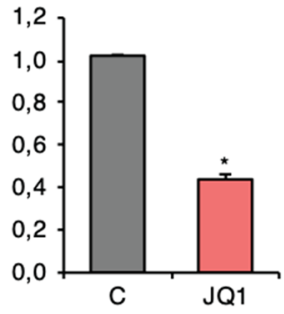

soxg

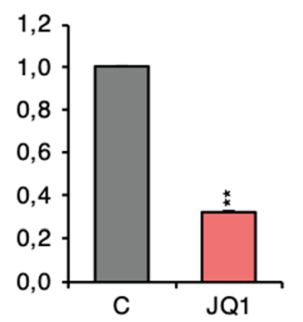

FSTL1

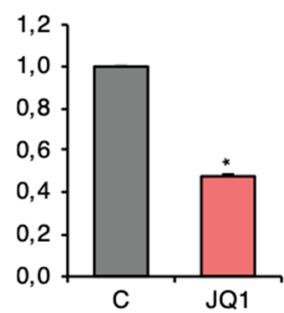

ITGA6

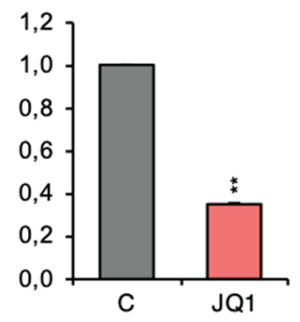

EZH2

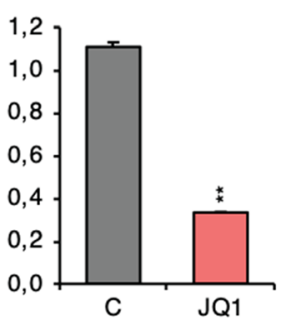

Sox2

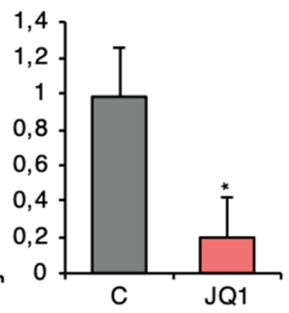

b

CD44

CD24
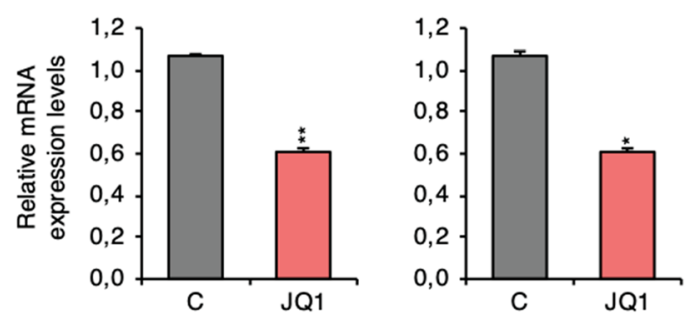

ALDH1A1
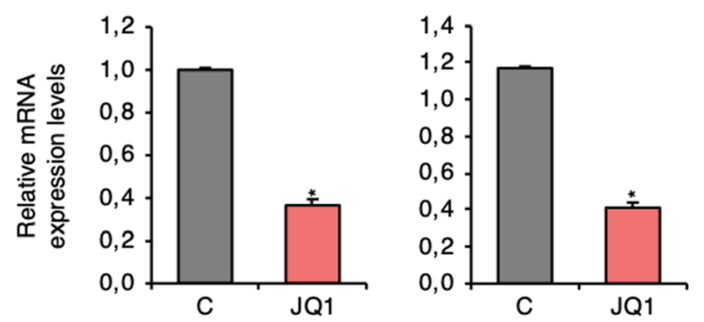

Fig. 4 JQ1 effects the expression of CSC markers in an in vivo preclinical model. a. MYC, ABCG2, ITGA6, EPCAM, SOX9, EZH2, GJA1, FSTL1 and SOX2 mRNA levels in JQ1-treated tumours compared to vehicle-treated tumours. b. CD44,CD24, ALDH1A1, KLF4 and
RUNX2 mRNA level changes observed upon JQ1 exposure. The qRTPCR conditions are described in material and methods. Student t-test: * $p<0,05, * * p<0,01$ and $* * * p<0,001$

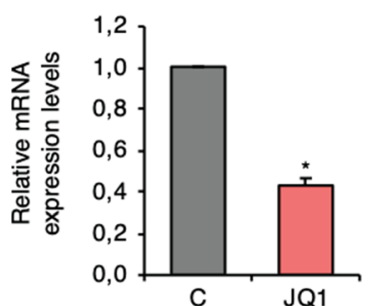

As mentioned above, targeting tumour stemness may be a good strategy to revert chemotherapy resistance and cancer recurrence. In this sense, some of the stemness-related genes identified in this study were able to predict relapse and a lack of response to chemotherapy in breast cancer patients. Among them, GJA1, CD24, EPCAM and SOX9 were found to be associated with a worse patient prognosis. Interestingly, we also found that combined expression of these four genes had a higher predictive potential than each gene individually. This may have clinical implications, as this 4-genes subpanel may potentially serve as a TNBC prognostic biomarker set.

On the other hand, using an online transcriptome-level validation tool for predictive biomarkers [52], we found that $A B C G 2$, associated with resistance to chemo- and radiotherapy [53] and RUNX2, which has been shown to chemosensitize breast cancer cells to neoadjuvant therapy [43], are more highly expressed in non-responder patients. Although not all the parameters analysed reached statistical significance, the observed trends could be corroborated using other probes available in the tool (data not shown). The association of $A B C G 2$ with resistance to therapy has been extensively confirmed [54]. Our results also confirm a role for RUNX2 as a potential predictive marker for chemotherapy response in TNBC. Although no significant association with either patient outcome or response to treatment was found for the rest of the genes contained in the stemness-related panel, evaluation of the expression levels of the whole stemness-panel, including all the 13 identified genes, might be of interest for the choice of treatment in patients with aggressive tumours.

Although BETi exhibit marked antitumor activities in TNBC, single treatment seems to have limited value for clinical cancer management. Conversely, combination therapy is gaining ground [55]. BETi have, for example, successfully been combined with kinase inhibitors $[13,56]$ and histone deacetylase inhibitors [57] in breast cancer, but its combination with chemotherapeutic agents has not been explored yet. Our data support the use of JQ1 in combination with other anticancer drugs to overcome therapy resistance. 
a

\begin{tabular}{|c|c|c|}
\hline \multicolumn{3}{|c|}{ K-M Plotter } \\
\hline Gene & HR & p-value \\
\hline EPCAM & $1,41(1,09-1,83)$ & 0,0089 \\
SOX9 & $1,41(1,09-1,81)$ & 0,0075 \\
GJA1 & $1,55(1,21-2)$ & 0,00057 \\
CD24 & $1,5(1,14-1,97)$ & 0,0034 \\
\hline
\end{tabular}

b

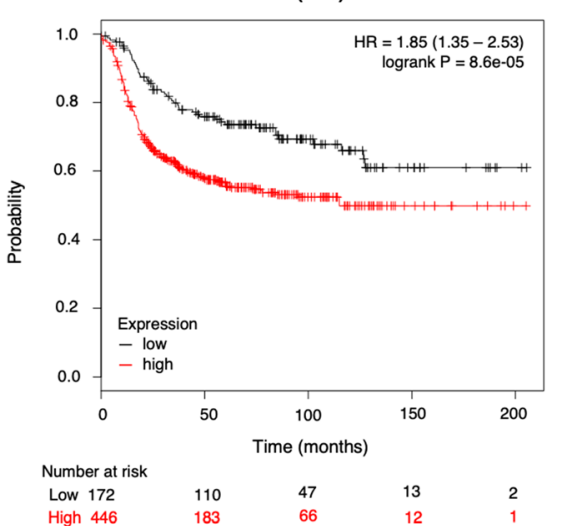

C
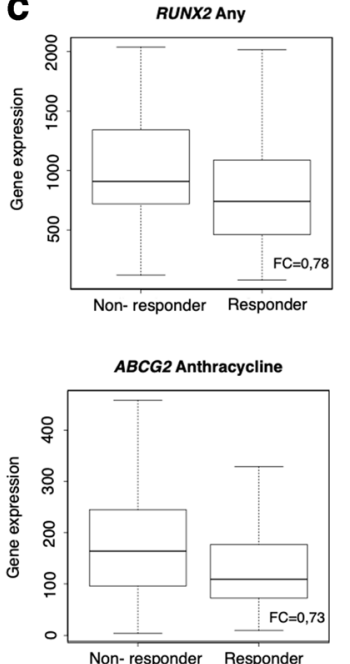
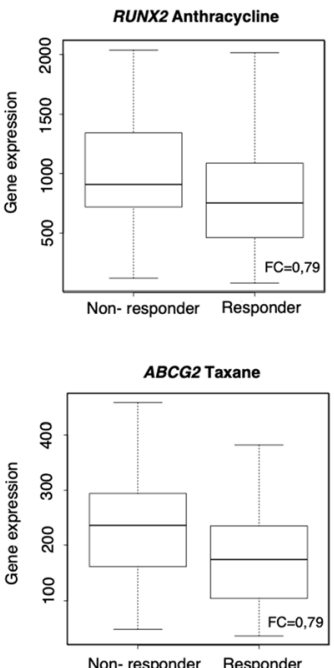

Fig. 5 JQ1-controlled genes EPCAM, SOX9, GJA1 and CD24 correlate with a poor prognosis, while $R U N X 2$ and $A B C G 2$ correlate with worse response to chemotherapy. a. The Kaplan-Meier plotter online tool was used to assess potential relations of JQ1 downregulated stemness-related genes and disease progression in basal-like breast cancer patients. Hazard Ratio (HR) and $p$ values of the EPCAM, SOX9, GJA1 and CD24 genes with a significant association a with worse relapse-free survival (RFS) are listed in the table. The cut-off values used to separate patients, referred to as best cut-off, were 8464, 2557, 3196 and 4844, respectively. b. Using the same tool, a combined analysis with the four genes to assess their link to patient prognosis was performed. Kaplan-Meier plots compare tumours with a low combined expression of these four genes with those exhibiting a high combined expression. HR and logrank $p$ values are indicated. The selected cut-off value $=3733.5$. c. Using transcriptomic data of 2108 patients with available treatment and response data, potential associations between $13 \mathrm{JQ1-controlled} \mathrm{stemness-related} \mathrm{genes} \mathrm{and} \mathrm{pa-}$ tient responses to chemotherapy were evaluated. The $A B C G 2$ and $R U N X 2$ mRNA expression levels in responder and non-responder patients, measured as RFS five years after chemotherapy completion, are shown for several chemotherapy regimens

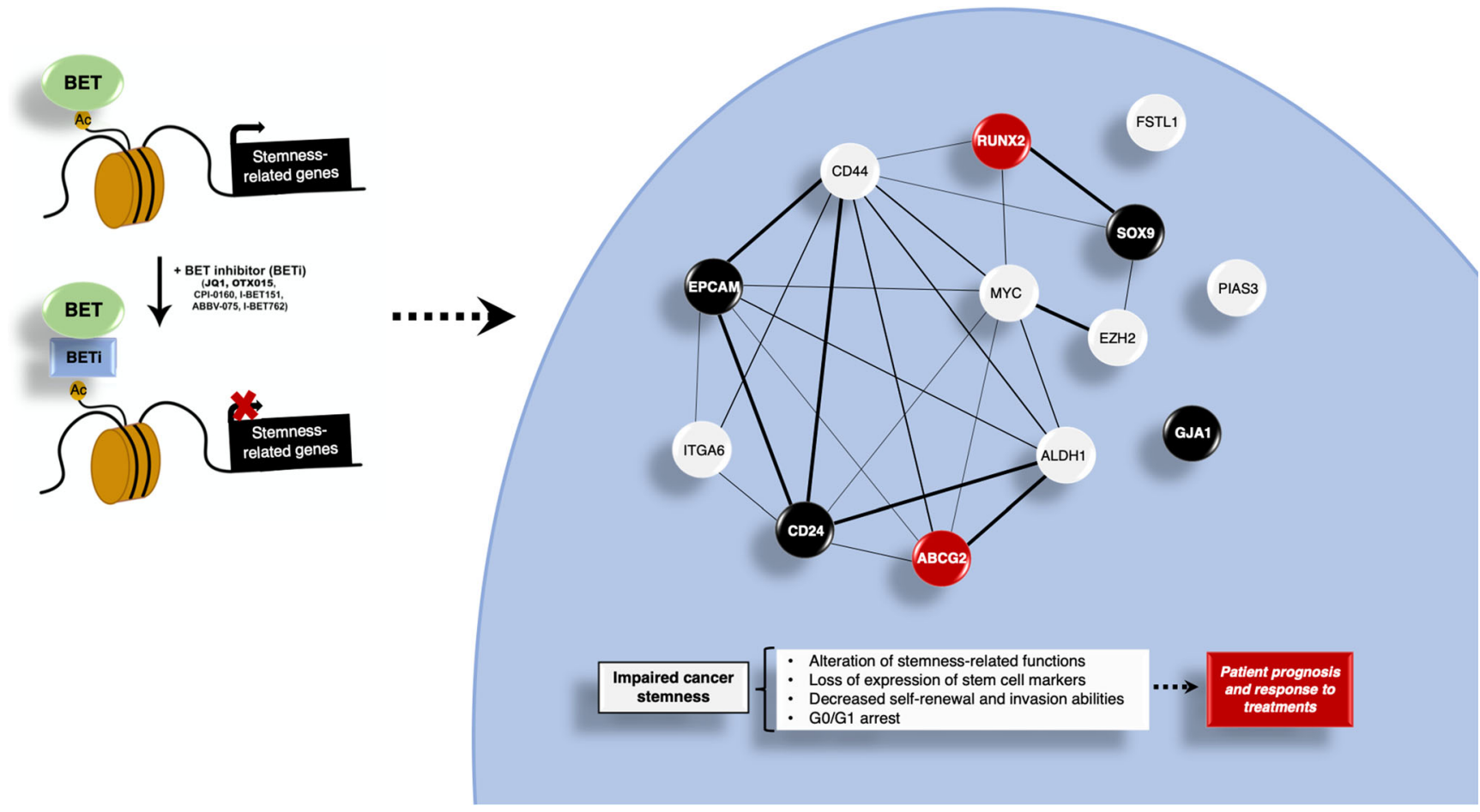

Fig. 6 JQ1 modifies stemness landscape in TNBC. JQ1 controls stemness-related genes in TNBC, which leads to impairment of cancer stemness. Thus, this BETi alters stemness-related functions, decreases self-renewal and invasion capabilities and provokes cell cycle arrest in G0/G1. The protein-protein interaction (PPI) map shows a high grade of interaction of the corresponding proteins (Average node degree: 3.69; Avg. local clustering coefficient: 0.51 ; PPI enrichment $p$-value:7.28e14). Black nodes indicate proteins encoded by genes associated with a poor RFS, and red nodes indicate proteins encoded by genes linked to poor response to conventional chemotherapy 


\section{Conclusions}

In summary, in this work we describe a novel function for the BETi JQ1 in TNBC as a stemness-targeting drug. We identified a 13-genes stemness signature which can be deregulated by JQ1 and may have potential as a biomarker for chemotherapy treatment response and patient prognosis in TNBC. Given the high level of interaction of the predicted gene signature network, and given the fact that the identification of biomarker-interacting maps and the discovery of drug targets are main goals of future medicine [58], JQ1 may be considered as a stemness interactome-based drug (Fig. 6). Altogether, our results support the idea that targeting the epigenome with JQ1, through its impact on stemness-related genes and features, may help to overcome TNBC resistance to standard therapy. This may have potential therapeutic implications with respect to the choice of anti-tumour agents for each patient, allowing more individualized TNBC treatment.

Acknowledgements We would like to thank the cancer associations AMUMA and ACEPAIN and their members for their support. We also thank Dr. Julie Dwyer for her help with editing the manuscript. In addition, we would like to acknowledge all members of the Translational Oncology group. In particular, we thank Fernando Andres-Pretel for this technical assistance.

Author contributions EMGM conceived the study and performed the original design of the experiments. LSO, MNC, SMG, CNJ, VSC, JPP and $\mathrm{MB}$ performed the experiments. SMG, BG, and MNC searched the data and performed the analysis. MNC, SMG, CNJ and EMGM prepared the figs. BG, AO and EMGM discussed the data. AO and EMGM drafted the manuscript. EMGM wrote the manuscript. All authors reviewed, included modifications and approved the final version of the manuscript.

Funding information Diputación de Albacete, AMUMA, and Junta de Comunidades de Castilla-La Mancha (SBPLY/19/180501/000173) (to EMGM). Instituto de Salud Carlos III (PI16/01121 and PI19/00808), ACEPAIN, Diputación de Albacete and CRIS Cancer Foundation (to AO). E.M.G.M. was funded by the implementation research program of the UCLM (UCLM resolution date: 31/07/2014), with a contract for accessing the Spanish System of Science, Technology and InnovationSecti (co-funded by the European Commission/FSE funds). SMG was funded by a fellowship from the Centro Regional de Investigaciones Biomédicas (CRIB). MB was supported by a Castilla-La Mancha Government regional grant for Biomedicine and Health Science Research ref.: II-2018 11. BG was supported by NVKP 16-1-20160037 and FIEK 16-1-2016-0005 grants of the National Research, Development and Innovation Office, Hungary.

Data Availability All data generated and/or analysed during the current study are available from the corresponding author upon reasonable request.

\section{Compliance with ethical standards}

Conflict of interest The authors declare that they have no conflict of interest.

Ethics statement All animal studies were carried out according to protocols approved by the ethics committee on animal experimentation of Castilla-
La Mancha University, in accordance with the requirements of the Institutional Guideline for Animal Experiments (Procedure PR-2017-03-07).

\section{Consent for publication Not applicable.}

Open Access This article is licensed under a Creative Commons Attribution 4.0 International License, which permits use, sharing, adaptation, distribution and reproduction in any medium or format, as long as you give appropriate credit to the original author(s) and the source, provide a link to the Creative Commons licence, and indicate if changes were made. The images or other third party material in this article are included in the article's Creative Commons licence, unless indicated otherwise in a credit line to the material. If material is not included in the article's Creative Commons licence and your intended use is not permitted by statutory regulation or exceeds the permitted use, you will need to obtain permission directly from the copyright holder. To view a copy of this licence, visit http://creativecommons.org/licenses/by/4.0/.

\section{References}

1. D. Hanahan, R.A. Weinberg, Hallmarks of Cancer: The next generation. Cell 144, 646-674 (2011)

2. L.L. Campbell, K. Polyak, Breast tumor heterogeneity: Cancer stem cells or clonal evolution? Cell Cycle 6, 2332-2338 (2007)

3. J. Kim, S.H. Orkin, Embryonic stem cell-specific signatures in cancer: Insights into genomic regulatory networks and implications for medicine. Genome Med 3, 75 (2011)

4. M. Dean, T. Fojo, S. Bates, Tumour stem cells and drug resistance. Nat Rev Cancer 5, 275-284 (2005)

5. S. Colak, J.P. Medema, Cancer stem cells - important players in tumor therapy resistance. FEBS J 281, 4779-4791 (2014)

6. T. Borovski, E. De Sousa, F. Melo, L. Vermeulen, J.P. Medema, Cancer stem cell niche: The place to be. Cancer Res 71, 634-639 (2011)

7. J. Monteiro, R. Fodde, Cancer stemness and metastasis: Therapeutic consequences and perspectives. Eur J Cancer 46, 1198-1203 (2010)

8. T.M. Malta, A. Sokolov, A.J. Gentles, T. Burzykowski, L. Poisson, J.N. Weinstein, B. Kamińska, J. Huelsken, L. Omberg, O. Gevaert, A. Colaprico, P. Czerwińska, S. Mazurek, L. Mishra, H. Heyn, A. Krasnitz, A.K. Godwin, A.J. Lazar, Machine learning identifies stemness features associated with oncogenic dedifferentiation. Cell 173, 338-354.e15 (2018)

9. M.A. Dawson, T. Kouzarides, Cancer epigenetics: From mechanism to therapy. Cell 150, 12-27 (2012)

10. E.N. Wainwright, P. Scaffidi, Epigenetics and Cancer stem cells: Unleashing, hijacking, and restricting cellular plasticity. Trends Cancer 3, 372-386 (2017)

11. N. Ahuja, A.R. Sharma, S.B. Baylin, Epigenetic therapeutics: A new weapon in the war against Cancer. Annu Rev Med 67, 73-89 (2016)

12. S. Genta, M.C. Pirosa, A. Stathis, BET and EZH2 inhibitors: Novel approaches for targeting Cancer. Curr Oncol Rep 21, 13 (2019)

13. C. Nieto-Jiménez, A. Alcaraz-Sanabria, J. Pérez-Peña, V. CorralesSánchez, G. Serrano-Heras, E.M. Galán-Moya, L. Serrano-Oviedo, J.C. Montero, M. Burgos, J. Llopis, A., Ocaña A. Pandiella, Targeting basal-like breast tumors with bromodomain and extraterminal domain (BET) and polo-like kinase inhibitors. Oncotarget 8, 19478-19490 (2017)

14. V. Sahai, A.J. Redig, K.A. Collier, F.D. Eckerdt, H.G. Munshi, Targeting BET bromodomain proteins in solid tumors. Oncotarget 7, 3997-54009 (2016) 
15. T. Braun, C. Gardin, Investigational BET bromodomain protein inhibitors in early stage clinical trials for acute myelogenous leukemia (AML). Expert Opin Investig Drugs 26, 803-811 (2017)

16. R.D. Chacón, M.V. Costanzo, Triple-negative breast cancer. Breast Cancer Res 12(Suppl 2), S3 (2010)

17. J.C. Montero, A. Esparís-Ogando, M.F. Re-Louhau, S. Seoane, M. Abad, R. Calero, A. Ocaña, A. Pandiella, Active kinase profiling, genetic and pharmacological data define mTOR as an important common target in triple-negative breast cancer. Oncogene 33, $148-156$ (2014)

18. A. Ocana, A. Pandiella, Targeting oncogenic vulnerabilities in triple negative breast cancer: biological bases and ongoing clinical studies. Oncotarget 8, 22218-22234 (2017)

19. M.H. Manjili, Tumor dormancy and relapse: From a natural byproduct of evolution to a disease state. Cancer Res 77, 2564 2569 (2017)

20. G. Bianchini, J.M. Balko, I.A. Mayer, M.E. Sanders, L. Gianni, Triple-negative breast cancer: Challenges and opportunities of a heterogeneous disease. Nat Rev Clin Oncol 13, 674 (2016)

21. W. Li, H. Ma, J. Zhang, L. Zhu, C. Wang, Y. Yang, Unraveling the roles of CD44/CD24 and ALDH1 as cancer stem cell markers in tumorigenesis and metastasis. Sci Rep 7, 13856 (2017)

22. E.-T. Verjans, J. Doijen, W. Luyten, B. Landuyt, L. Schoofs, Threedimensional cell culture models for anticancer drug screening: Worth the effort? J Cell Physiol 233, 2993-3003 (2018)

23. Z. Mihály, M. Kormos, A. Lánczky, M. Dank, J. Budczies, M.A. Szász, B. Györffy, A meta-analysis of gene expression-based biomarkers predicting outcome after tamoxifen treatment in breast cancer. Breast Cancer Res Treat 140, 219-232 (2013)

24. M.M. Gottesman, T. Fojo, S.E. Bates, Multidrug resistance in cancer: Role of ATP-dependent transporters. Nat Rev Cancer 2, 48-58 (2002)

25. J.P. Pinto, R.K. Kalathur, D.V. Oliveira, T. Barata, R.S.R. Machado, S. Machado, I. Pacheco-Leyva, I. Duarte, M.E. Futschik, StemChecker: A web-based tool to discover and explore stemness signatures in gene sets. Nucleic Acids Res 43, W72-W77 (2015)

26. J. Zuber, J. Shi, E. Wang, A.R. Rappaport, H. Herrmann, E.A. Sison, D. Magoon, J. Qi, K. Blatt, M. Wunderlich, M.J. Taylor, C. Johns, A. Chicas, J.C. Mulloy, S.C. Kogan, P. Brown, P. Valent, J.E. Bradner, S.W. Lowe, C.R. Vakoc, RNAi screen identifies Brd4 as a therapeutic target in acute myeloid leukaemia. Nature 478, 524-528 (2011)

27. Y. Wu, Y. Wang, P. Diao, W. Zhang, J. Li, H. Ge, Y. Song, Z. Li, D. Wang, L. Liu, H. Jiang, J. Cheng, Therapeutic targeting of BRD4 in head neck squamous cell carcinoma. Theranostics 9, 1777-1793 (2019)

28. S. Venataraman, I. Alimova, P. Harris, D.K. Birks, I. Balakrishnan, M. Remke, M.D. Taylor, M. Handler, N.K. Foreman, R. Vibhakar, Inhibition of BRD4 attenuates tumor cell self-renewal and suppresses stem cell signaling in MYC driven medulloblastoma. Oncotarget 5, 2355-2371 (2014)

29. S. Shu, C.Y. Lin, H.H. He, R.M. Witwicki, D.P. Tabassum, J.M. Roberts, M. Janiszewska, S.J. Huh, Y. Liang, J. Ryan, E. Doherty, H. Mohammed, H. Guo, D.G. Stover, M.B. Ekram, J. Brown, C. D'Santos, I.E. Krop, D. Dillon, M. McKeown, C. Ott, J. Qi, M. Ni, P.K. Rao, M. Duarte, S.Y. Wu, C.M. Chiang, L. Anders, R.A. Young, E. Winer, A. Letai, W.T. Barry, J.S. Carroll, H. Long, M. Brown, X.S. Liu, C.A. Meyer, J.E. Bradner, K. Polyak, Response and resistance to BET bromodomain inhibitors in triple-negative breast cancer. Nature 529, 413-417 (2016)

30. J.M. Sahni, S.S. Gayle, K.L.W. Bonk, L.C. Vite, J.L. Yori, B. Webb, E.K. Ramos, D.D. Seachrist, M.D. Landis, J.C. Chang, J.E. Bradner, R.A. Keri, Bromodomain and Extraterminal protein inhibition blocks growth of triple-negative breast cancers through the suppression of Aurora kinases. J Biol Chem 291, 23756-23768 (2016)
31. J.M. Sahni, S.S. Gayle, B.M. Webb, K.L. Weber-Bonk, D.D. Seachrist, S. Singh, S.T. Sizemore, N.A. Restrepo, G. Bebek, P.C. Scacheri, V. Varadan, M.K. Summers, R.A. Keri, Mitotic vulnerability in triple-negative breast Cancer associated with LIN9 is targetable with BET inhibitors. Cancer Res 77, 5395-5408 (2017)

32. G. Andrieu, A.H. Tran, K.J. Strissel, G.V. Denis, BRD4 regulates breast Cancer dissemination through Jagged1/Notch1 signaling. Cancer Res 76, 6555-6567 (2016)

33. J.S. Zawistowski, S.M. Bevill, D.R. Goulet, T.J. Stuhlmiller, A.S. Beltran, J.F. Olivares-Quintero, D. Singh, N. Sciaky, J.S. Parker, N.U. Rashid, X. Chen, J.S. Duncan, M.C. Whittle, S.P. Angus, S.H. Velarde, B.T. Golitz, X. He, C. Santos, D.B. Darr, K. Gallagher, L.M. Graves, C.M. Perou, L.A. Carey, H.S. Earp, G.L. Johnson, Enhancer remodeling during adaptive bypass to MEK inhibition is attenuated by pharmacologic targeting of the P-TEFb complex. Cancer Discov 7, 302-321 (2017)

34. G.P. Andrieu, G.V. Denis, BET proteins exhibit transcriptional and functional opposition in the epithelial-to-Mesenchymal transition. Mol Cancer Res 16, 580-586 (2018)

35. A.R. Green, M.A. Aleskandarany, D. Agarwal, S. Elsheikh, C.C. Nolan, M. Diez-Rodriguez, R.D. Macmillan, G.R. Ball, C. Caldas, S. Madhusudan, I.O. Ellis, E.A. Rakha, MYC functions are specific in biological subtypes of breast cancer and confers resistance to endocrine therapy in luminal tumours. Br J Cancer 114, 917-928 (2016)

36. S. Guo, X. Li, J. Rohr, Y. Wang, S. Ma, P. Chen, Z. Wang, EZH2 overexpression in different immunophenotypes of breast carcinoma and association with clinicopathologic features. Diagn Pathol 11, 41 (2016)

37. M. Nuncia-Cantarero, S. Martinez-Canales, F. Andrés-Pretel, G. Santpere, A. Ocaña, E.M. Galan-Moya, Functional transcriptomic annotation and protein-protein interaction network analysis identify NEK2, BIRC5, and TOP2A as potential targets in obese patients with luminal a breast cancer. Breast Cancer Res Treat 168, 613-623 (2018)

38. G. Sashida, C. Wang, T. Tomioka, M. Oshima, K. Aoyama, A. Kanai, M. Mochizuki-Kashio, H. Harada, K. Shimoda, A. Iwama, The loss of Ezh2 drives the pathogenesis of myelofibrosis and sensitizes tumor-initiating cells to bromodomain inhibition. J Exp Med 213, 1459-1477 (2016)

39. M.A. Voronkova, S. Luanpitpong,1 L.W. Rojanasaku, V. Castranova, C.Z. Dinu, H. Riedel, Y. Rojanasakul, SOX9 regulates cancer stem-like properties and metastatic potential of singlewalled carbon nanotube-exposed cells. Sci Rep 7, 11653 (2017)

40. Y. Wen, J. Cai, Y. Hou, Z. Huang, Z. Wang, Role of EZH2 in cancer stem cells: from biological insight to a therapeutic target. Oncotarget 8, 37974-37990 (2017)

41. N. Ferrari, L. McDonald, J.S. Morris, E.R. Cameron, K. Blyth, RUNX2 in mammary gland development and breast cancer. J Cell Physiol 228, 1137-1142 (2013)

42. X.-Q. Li, J.-T. Lu, C.-C. Tan, Q.-S. Wang, Y.-M. Feng, RUNX2 promotes breast cancer bone metastasis by increasing integrin $\alpha 5$ mediated colonization. Cancer Lett 380, 78-86 (2016)

43. V. Sancisi, G. Manzotti, M. Gugnoni, T. Rossi, G. Gandolfi, G. Gobbi, et al., RUNX2 expression in thyroid and breast cancer requires the cooperation of three non-redundant enhancers under the control of BRD4 and c-JUN. Nucleic Acids Res 45, 11249-11267 (2017)

44. M. Munz, P.A. Baeuerle, O. Gires, The emerging role of EpCAM in Cancer and stem cell signaling. Cancer Res 69, 5627-5629 (2009)

45. T. Hiraga, S. Ito, H. Nakamura, EpCAM expression in breast cancer cells is associated with enhanced bone metastasis formation: EpCAM expression is associated with bone metastases of breast cancer. Int J Cancer 138, 1698-1708 (2016) 
46. T. Hu, R. Zhou, Y. Zhao, G. Wu, Integrin $\alpha 6 /$ Akt/Erk signaling is essential for human breast cancer resistance to radiotherapy. Sci Rep 6, 3376 (2016)

47. B. Mostert, J. Kraan, A.M. Sieuwerts, P. van der Spoel, J. Bolt-de Vries, Prager-van der Smissen WJC, et al. CD49f-based selection of circulating tumor cells (CTCs) improves detection across breast cancer subtypes. Cancer Lett 319, 49-55 (2012)

48. S.L. Phillips, C.B. Williams, J.N. Zambrano, C.J. Williams, E.S. Yeh, Connexin 43 in the development and progression of breast cancer: What's the connection? (review). Int J Oncol 51, 10051013 (2017)

49. M.C.-C. Lau, K.Y. Ng, T.L. Wong, M. Tong, T.K. Lee, X.-Y. Ming, et al., FSTL1 promotes metastasis and Chemoresistance in esophageal squamous cell carcinoma through NFKB-BMP signaling cross-talk. Cancer Res 77, 5886-5899 (2017)

50. J.-J. Qin, L. Yan, J. Zhang, W-D. Zhang, STAT3 as a potential therapeutic target in triple negative breast cancer: a systematic review. J Exp Clin Cancer Res 38, 195 (2019)

51. K. Banerjee, H. Resat, Constitutive activation of STAT3 in breast cancer cells: A review: Constitutive STAT3 activation in breast cancer. Int J Cancer 138, 2570-2578 (2016)

52. J.T. Fekete, B. Győrffy, ROCplot.org: Validating predictive biomarkers of chemotherapy/hormonal therapy/anti-HER2 therapy using transcriptomic data of 3,104 breast cancer patients. Int $\mathrm{J}$ Cancer 145, 3140-3151 (2019)
53. J. Hu, J. Li, X. Yue, J. Wang, J. Liu, L. Sun, D. Kong, Expression of the cancer stem cell markers ABCG2 and OCT-4 in right-sided colon cancer predicts recurrence and poor outcomes. Oncotarget 8, 28463-28470 (2017)

54. Q. Mao, J.D. Unadkat, Role of the breast Cancer resistance protein (BCRP/ABCG2) in drug transport-An update. AAPS J 17, 65-82 (2015)

55. A. Alqahtani, K. Choucair, M. Ashraf, D.M. Hammouda, A. Alloghbi, T. Khan, N. Senzer, J. Nemunaitis, Bromodomain and extra-terminal motif inhibitors: a review of preclinical and clinical advances in cancer therapy. Future Sci OA 5, FSO372 (2019)

56. E.E. Stratikopoulos, M. Dendy, M. Szabolcs, A.J. Khaykin, C. Lefebvre, M.-M. Zhou, R. Parsons, Kinase and BET inhibitors together clamp inhibition of PI3K signaling and overcome resistance to therapy. Cancer Cell 27, 837-851 (2015)

57. G. Borbely, L.-A. Haldosen, K. Dahlman-Wright, C. Zhao, Induction of USP17 by combining BET and HDAC inhibitors in breast cancer cells. Oncotarget 6, 33623-33635 (2015)

58. D.H. Roukos, Disrupting cancer cells' biocircuits with interactomebased drugs: Is 'clinical' innovation realistic? Expert Rev Proteomics 9, 349-353 (2012)

Publisher's note Springer Nature remains neutral with regard to jurisdictional claims in published maps and institutional affiliations. 